\title{
On the Inverse Problem for Quantum Graphs with One Cycle
}

\author{
P. KURASOV* \\ Dept. of Mathematics, LTH, Lund Univ., Box 118, 22100 Lund, Sweden, \\ Dept. of Mathematics, Stockholm Univ., 106 91, Stockholm, Sweden \\ and \\ Dept. of Physics, St. Petersburg Univ., 198904 St. Peterhof, Russia
}

\begin{abstract}
Quantum graphs having one cycle are considered. It is shown that if the cycle contains at least three vertices, then the potential on the graph can be uniquely reconstructed from the corresponding Titchmarsh-Weyl function (Dirichlet-to-Neumann map) associated with graph's boundary, provided certain non-resonant conditions are satisfied.
\end{abstract}

PACS numbers: 03.65.Nk, 73.63.-b, 85.35.-p

\section{Introduction}

Investigation of quantum graphs - differential operators on metric graphs - is a very important direction of research in modern mathematical physics. Its importance is explained by possible applications of such models in nanophysics and studies of chaotic phenomena. If spectral and scattering properties of such systems are rather well-understood (see recent reviews in [1-5]), the corresponding inverse problems appear to be rather difficult. Let us note that solution of the inverse problem consists in reconstructing:

- the metric graph;

- the differential operator (or some of its coefficients, like potential in the Schrödinger equation);

- the matching conditions at the vertices (coupling together different edges and making the operator self-adjoint).

The first inverse problem is relatively easy to analyze, but numerous counterexamples show that pretty often solution is not unique [6-10]. For general discussion of the current status of inverse problems for graphs we refer to [11] (see also [12-15]). The main subject of the current paper is the second inverse problem in its classical form: recovering of the potential in the Schrödinger equation. This problem has been solved in the case of trees [16-24]. During these investigations it became clear that the Titchmarsh-Weyl (TW) matrix function associated with all boundary points of the graph may play the role

\footnotetext{
* e-mail: kurasov@maths.lth.se
}

of the spectral data for such problems ${ }^{\dagger}$. This function is sometimes called by Dirichlet-to-Neumann map, since it connects Dirichlet and Neumann data for solutions of the eigenfunction equation. It is a matrix Nevanlinna function [25] and is closely related to the multichannel scattering [26].

We are going to consider graphs with cycles. Very few results are available for such systems and it appears natural to start our studies by looking at graphs with just one cycle. The inverse problem for trees can be solved using e.g. the boundary control method [27, 20,24] allowing one to reconstruct potential locally starting from the graph's boundary. If the graph has at least one cycle, then the potential on the cycle cannot be reconstructed using local methods: calculation of the potential requires consideration of the whole cycle at once. Moreover this problem may not have a unique solution [28, 7, 29]. It appeared that this inverse problem is closely related to the inverse problem for periodic one-dimensional Schrödinger operators and we are going to use methods developed in [30-34]. These methods have been applied in [11] to analyze simplest graphs with one cycle. It appears that the number of vertices on the cycle plays a very important role ${ }^{\ddagger}$. In the case of graphs with loops (cycles attached by its end points to just one vertex) potential cannot always be reconstructed uniquely. The same holds true in the case of cycles containing just two vertices. It was sug-

\footnotetext{
$\dagger$ For general definitions of graph's boundary and corresponding Titchmarsh-Weyl function see [11]. In the special case under consideration these objects are rigorously defined in Sect. 2.

¥ In what follows speaking about the number of vertices on the cycle we are going to have in mind only vertices that join together at least three edges. The vertices joining together just two vertices can be removed, since only standard matching conditions
} (2.1) are considered in this paper. 
gested in [11] to take into consideration magnetic fields in order to extract additional information on the system. It was shown that for graphs having one cycle which is not a loop the knowledge of the Titchmarsh-Weyl function for two different values of the magnetic flux through the cycle allows one to reconstruct potential uniquely, provided certain non-resonance conditions are hold true. But this approach is based on detailed studies of graphs having one cycle with just two vertices. It was conjectured that if the number of vertices on the cycle is larger than two, then the potential may always be reconstructed without any help of the magnetic field. Another conjecture concerns the possibility to reconstruct potential in the case of graphs completely covered by shortest paths connecting all boundary points. In order to show that these conjectures in general do not hold we decided to study the case of graphs having one cycle with three vertices. It is proven that the reconstruction is unique only if the system has no resonance (described in Sect. 3). It is also shown that if the system has a resonance, then the inverse problem may have multiple solutions.

Our result is closely related to the theorem proven by Yurko [29], who showed that the inverse problem for such graphs has a unique solution, provided the set of spectral data is extended by adding a certain sequence of signs. Such extension of the set of scattering data is essential, since it allows one to recover the potential even for loops. It also appears problematic to obtain such sequence in a realistic experiment. Moreover it is not clear whether this sequence is really necessary. Our results imply that under certain explicit conditions satisfied for almost all potentials, the reconstruction can be done using just the Titchmarsh-Weyl function and therefore the infinite sequence of signs may be redundant.

\section{Cycle with three contact points}

Consider the graph $\Gamma$ formed by one cycle with three edges attached to it (see Fig. 1). The corresponding Schrödinger operator $L$ is defined on the domain $\operatorname{Dom}(L)$ of functions from $\oplus \sum_{j=1}^{6} W_{2}^{2}\left[x_{2 j-1}, x_{2 j}\right] \subset L_{2}(\Gamma)$ satisfying standard matching conditions at the three internal vertices $V_{1}=\left\{x_{1}, x_{6}, x_{7}\right\}, V_{2}=\left\{x_{2}, x_{3}, x_{9}\right\}$, and $V_{3}=\left\{x_{4}, x_{5}, x_{11}\right\}$ :

$$
\left\{\begin{array}{l}
\psi\left(x_{i}\right)=\psi\left(x_{j}\right), x_{i}, x_{j} \in V_{m}, \quad m=1,2,3, \\
\sum_{x_{j} \in V_{m}} \partial_{n} \psi\left(x_{j}\right)=0,
\end{array}\right.
$$

and Dirichlet boundary conditions at the boundary vertices $V_{4}=\left\{x_{8}\right\}, V_{5}=\left\{x_{10}\right\}$, and $V_{6}=\left\{x_{12}\right\}$ :

$$
\psi\left(x_{j}\right)=0, \quad j=8,10,12 .
$$

Here $\partial_{n} \psi\left(x_{j}\right)$ denotes the normal derivative of the function $u$ in the direction from the corresponding vertex. Depending on whether $x_{j}$ is the left or the right end point of the corresponding interval, $\partial_{n} \psi\left(x_{j}\right)$ is equal either to $\psi^{\prime}\left(x_{j}\right)$ or to $-\psi^{\prime}\left(x_{j}\right)$.

The action of the operator $L$ is given by the Schrödinger differential expression

$$
L \psi=-\psi^{\prime \prime}+q(x) \psi,
$$

where $q(x) \in L_{2}(\Gamma)$ is a real valued potential. The operator $L$ so defined is self-adjoint in the Hilbert space $L_{2}(\Gamma)$.

With the differential operator $L$ we associate so-called Titchmarsh-Weyl (matrix) function $\boldsymbol{M}(\lambda)$ (denoted by TW-function in what follows). Let $\psi$ be a solution to the differential equation

$$
-\psi^{\prime \prime}+q(x) \psi=\lambda \psi, \quad \Im \lambda>0,
$$

satisfying standard matching conditions (2.1) at all internal vertices and prescribed boundary values $\psi\left(x_{8}\right), \psi\left(x_{10}\right), \psi\left(x_{12}\right)$ at the boundary vertices. Such solution always exists and is unique, since otherwise the self-adjoint operator $L$ would have a nonreal eigenvalue.

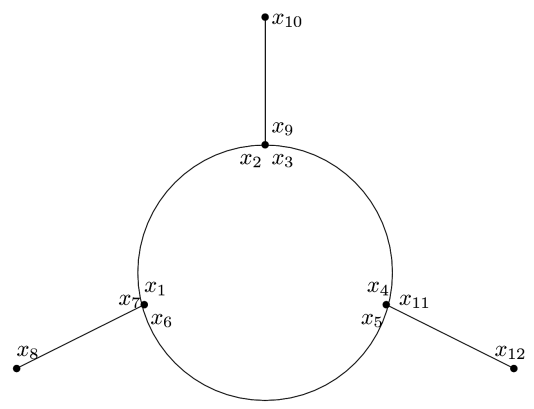

Fig. 1. Graph $\Gamma$. Ring with three wires.

It appears that the TW-function for the graph $\Gamma$ in general does not determine the potential $q$ on its kernel, i.e. on the cycle obtained from $\Gamma$ by cutting off all three boundary edges $\left[x_{7}, x_{8}\right],\left[x_{9}, x_{11}\right]$ and $\left[x_{11}, x_{12}\right]$. From our point of view the best way to describe sufficient conditions which guarantee unique solution to the inverse problem is using the notion of resonance introduced in the following section.

\section{Kernel resonance}

Let us consider the following definition:

Definition 1. We are going to say that the Schrödinger operator on the graph $\Gamma$ has a resonance with the energy $E$ if and only if the Dirichlet-Dirichlet spectra of the Sturm-Liouville operators on the intervals $\left[x_{1}, x_{2}\right],\left[x_{3}, x_{4}\right]$, and $\left[x_{5}, x_{6}\right]$ contain one and the same eigenvalue $E^{\S}$.

The following two lemmas characterize the notion of the resonance.

Lemma 1. Assume that the operator $L$ in $L_{2}(\Gamma)$ has an eigenfunction supported by the kernel of $\Gamma$, then the system has a resonance with the same energy.

\footnotetext{
$\S$ Using the transfer matrices $T^{m}$ for the intervals $\left[x_{2 m-1}, x_{2 m}\right]$ (defined by (4.2)) the resonance condition can be written in the form $t_{12}^{1}(E)=t_{12}^{2}(E)=t_{12}^{3}(E)=0$.
} 
The proof is almost identical to the proof of Lemma 7.1 from [11].

To formulate the second lemma we shall need the notion of the scattering matrix associated with the graph $\Gamma$. Consider the extended graph $\Gamma^{\text {ext }}$ (see Fig. 2) obtained from $\Gamma$ by attaching semi-infinite intervals to its boundary vertices $x_{8}, x_{10}$ and $x_{12}$. The potential $q$ is extended to the whole $\Gamma^{\text {ext }}$ by putting $\left.q\right|_{\Gamma^{\text {ext }} \backslash \Gamma} \equiv 0$. Then the scattering matrix $\boldsymbol{S}(\lambda)$ for the Schrödinger operator on $\Gamma^{\text {ext }}$ and the TW-function for the graph $\Gamma$ are related as follows (see [11] for details):

$$
\boldsymbol{S}(\lambda)=\frac{\mathrm{i} k-\boldsymbol{M}(\lambda)}{\mathrm{i} k+\boldsymbol{M}(\lambda)} .
$$

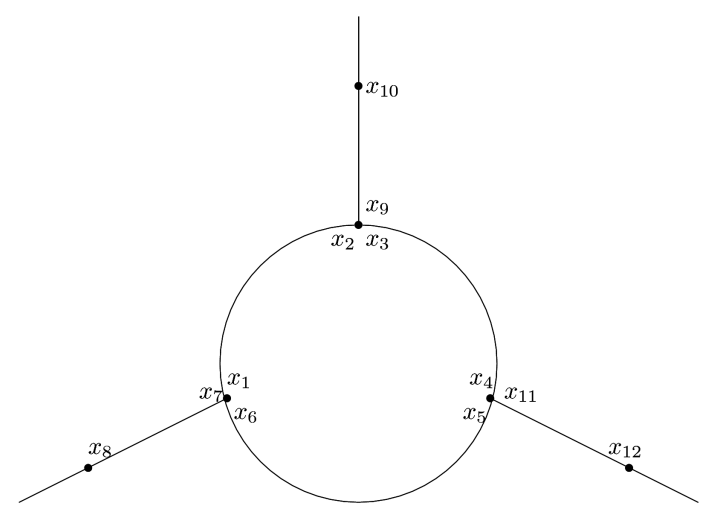

Fig. 2. The extended graph $\Gamma^{\mathrm{ext}}$.

Lemma 2. Assume that the operator $L$ has a resonance with the energy $E$, then either the operator $L$ has an eigenfunction with the same energy supported by the kernel of the graph (equivalently, has support separated from the boundary) or the corresponding scattering matrix $\boldsymbol{S}(E)$ is diagonal.

Proof. The proof is again similar to the proof of the corresponding lemma in [11] (Lemma 7.2). Assume that the system has a resonance with the energy $E$. Then using the eigenfunctions of the Sturm-Liouville operators on $\left[x_{1}, x_{2}\right],\left[x_{3}, x_{4}\right]$, and $\left[x_{5}, x_{6}\right]$ one may construct a not identically equal to zero function $f_{\text {ker }}$, which is equal to zero at $V_{1}, V_{2}$ and $V_{3}$ and has continuous derivative everywhere on the cycle (including vertices $V_{2}$ and $V_{3}$ ) but may be not at the vertex $V_{1}$. Consider the following two possible cases:

1. If $f_{\text {ker }}$ has continuous first derivative at $V_{1}$, then extending the function by zero to the rest of $\Gamma$ we get an eigenfunction of $L$ supported by the kernel of $\Gamma$.

2. If the first derivative of $f_{\text {ker }}$ is not continuous at $V_{1}$, then consider the unique (up to a multiplier) function $f_{\text {scat }}$ which solves (2.4) on the interval $\left[x_{7}, \infty\right)$ and satisfies Dirichlet boundary condition at $x_{7}$. Now using the functions $f_{\text {ker }}$ and $f_{\text {scat }}$ one may combine a scattered wave equal to zero on $\left[x_{9}, \infty\right)$ and $\left[x_{11}, \infty\right)$ and satisfying matching conditions (2.1) at all vertices. It follows that the corresponding reflection coefficient has absolute value 1 .

The other two wires can be considered in a similar way leading to the conclusion that the system either has an eigenfunction supported by its kernel, or the corresponding scattering matrix is diagonal at this energy.

Summing up we would like to underline that the notion of the resonance is closely related to eigenfunctions supported by the kernel of the graph and to quantum blockade. The diagonality of the scattering matrix at a certain energy implies that plane waves with this energy do not penetrate through the system but are completely reflected by it.

\section{Solution of the inverse problem for systems without resonances}

The TW (matrix) function associated with the graph $\Gamma$ can easily be calculated using TW-functions associated with each interval $\left[x_{2 m-1}, x_{2 m}\right], m=1, \ldots, 6$. In [11] it was proven that the potential on the boundary edges, i.e. on the edges $\left[x_{7}, x_{8}\right],\left[x_{9}, x_{10}\right],\left[x_{11}, x_{12}\right]$ in our case, can be reconstructed using the boundary control method described for example in [27, 24, 35]. Therefore the problem we are going to discuss here is how to reconstruct the kernel of the graph and potential $q$ on it. The kernel of the graph is nothing else than the cycle formed by the intervals $\left[x_{1}, x_{2}\right],\left[x_{3}, x_{4}\right],\left[x_{5}, x_{6}\right]$ coupled together in a circular way. The kernel has three contact points $x_{7}, x_{9}, x_{11}$ and the corresponding TW function is a $3 \times 3$ matrix function connecting together the boundary values of any solution to the differential equation (2.4) satisfying the matching conditions (2.1):

$$
\left(\begin{array}{c}
\psi^{\prime}\left(x_{7}\right) \\
\psi^{\prime}\left(x_{9}\right) \\
\psi^{\prime}\left(x_{11}\right)
\end{array}\right)=-\boldsymbol{M}_{\mathrm{ker}}(\lambda)\left(\begin{array}{c}
\psi\left(x_{7}\right) \\
\psi\left(x_{9}\right) \\
\psi\left(x_{11}\right)
\end{array}\right) .
$$

This function can be calculated in terms of the transfer matrices $T^{m}$ associated with the intervals $\left[x_{2 m-1}, x_{2 m}\right]$, $m=1,2,3$ :

$$
\begin{aligned}
& \left(\begin{array}{c}
\psi\left(x_{2 m}\right) \\
\psi^{\prime}\left(x_{2 m}\right)
\end{array}\right)=T^{m}\left(\begin{array}{c}
\psi\left(x_{2 m-1}\right) \\
\psi^{\prime}\left(x_{2 m-1}\right)
\end{array}\right), \\
& T^{m}=\left(\begin{array}{cc}
t_{11}^{m} & t_{12}^{m} \\
t_{21}^{m} & t_{22}^{m}
\end{array}\right),
\end{aligned}
$$

where $\psi$ is any solution to $(2.4)$ on $\left[x_{2 m-1}, x_{2 m}\right]$. The matrices $T^{m}$ have very special analytic properties and uniquely determine the potential $q$ on the corresponding interval. In what follows it will be convenient to consider $t_{i j}^{m}$ as functions of the spectral parameter $k, k^{2}=\lambda$.

To calculate the TW-matrix we put down the following system of equations: 


$$
\left\{\begin{array}{l}
\psi\left(x_{7}\right)=\psi\left(x_{1}\right)=t_{11}^{3} \psi\left(x_{5}\right)+t_{12}^{3} \psi^{\prime}\left(x_{5}\right), \\
\psi\left(x_{9}\right)=\psi\left(x_{3}\right)=t_{11}^{1} \psi\left(x_{1}\right)+t_{12}^{1} \psi^{\prime}\left(x_{1}\right), \\
\psi\left(x_{11}\right)=\psi\left(x_{5}\right)=t_{11}^{2} \psi\left(x_{3}\right)+t_{12}^{2} \psi^{\prime}\left(x_{3}\right), \\
\psi^{\prime}\left(x_{7}\right)+\psi^{\prime}\left(x_{1}\right)-\left[t_{21}^{3} \psi\left(x_{5}\right)+t_{22}^{3} \psi^{\prime}\left(x_{5}\right)\right]=0, \\
\psi^{\prime}\left(x_{9}\right)+\psi^{\prime}\left(x_{3}\right)-\left[t_{21}^{1} \psi\left(x_{1}\right)+t_{22}^{1} \psi^{\prime}\left(x_{1}\right)\right]=0, \\
\psi^{\prime}\left(x_{11}\right)+\psi^{\prime}\left(x_{5}\right)-\left[t_{21}^{2} \psi\left(x_{3}\right)+t_{22}^{2} \psi^{\prime}\left(x_{3}\right)\right]=0 .
\end{array}\right.
$$

The first three lines express the fact that the function $\psi$ is continuous at the vertices and the last three equations describe the balance of the first derivatives at the vertices (the second equation in (2.1)). In addition we have expressed the values $\psi\left(x_{2 m}\right), \psi^{\prime}\left(x_{2 m}\right), m=1,2,3$ using the transfer matrices. The system (4.3) consists of totally 9 linear equations connecting 12 variables. Excluding the variables $\psi\left(x_{2 m-1}\right), \psi^{\prime}\left(x_{2 m-1}\right), m=1,2,3$ one easily calculates the corresponding TW-function

$$
\boldsymbol{M}_{\mathrm{ker}}(\lambda)=\left(\begin{array}{ccc}
-\frac{t_{11}^{1} t_{12}^{3}+t_{12}^{1} t_{22}^{3}}{t_{12}^{1} t_{12}^{3}} & \left(t_{12}^{1}\right)^{-1} & \left(t_{12}^{3}\right)^{-1} \\
\left(t_{12}^{1}\right)^{-1} & -\frac{t_{11}^{2} t_{12}^{1}+t_{12}^{2} t_{22}^{1}}{t_{12}^{2} t_{12}^{1}} & \left(t_{12}^{2}\right)^{-1} \\
\left(t_{12}^{3}\right)^{-1} & \left(t_{12}^{2}\right)^{-1} & -\frac{t_{11}^{3} t_{12}^{2}+t_{12}^{3} t_{22}^{2}}{t_{12}^{3} t_{12}^{2}}
\end{array}\right)
$$

or in other notations

$$
\begin{aligned}
& \boldsymbol{M}_{\mathrm{ker}}(\lambda) \\
& =\left(\begin{array}{ccc}
-\frac{\left(T^{1} T^{3}\right)_{12}}{t_{12}^{1} t_{12}^{3}} & \left(t_{12}^{1}\right)^{-1} & \left(t_{12}^{3}\right)^{-1} \\
\left(t_{12}^{1}\right)^{-1} & -\frac{\left(T^{2} T^{1}\right)_{12}}{t_{12}^{2} t_{12}^{1}} & \left(t_{12}^{2}\right)^{-1} \\
\left(t_{12}^{3}\right)^{-1} & \left(t_{12}^{2}\right)^{-1} & -\frac{\left(T^{3} T^{2}\right)_{12}}{t_{12}^{3} t_{12}^{2}}
\end{array}\right) .
\end{aligned}
$$

Theorem 1. Let $\Gamma$ be a metric graph with Euler characteristic zero (i.e. having just one cycle) and the kernel having three contact points. Let $L=-\frac{\mathrm{d}^{2}}{\mathrm{~d} x^{2}}+q$ be a Schrödinger operator in $L_{2}(\Gamma)$ determined by a real potential $q \in L_{2}(\Gamma)$ and matching/boundary conditions (2.1) and (2.2). Assume that there is no resonance, then the Titchmarsh-Weyl function associated with all boundary points of $\Gamma$ determines the metric graph $\Gamma$ and the real potential $q$ uniquely.

Proof. Every (finite) graph $\Gamma$ can be seen as its kernel with several branches attached to it [11]. In the case under consideration the kernel is just one cycle and the number of branches is equal to three. As is proven in $[24,11]$, the boundary control method allows one to reconstruct all the branches and potentials on them using the corresponding TW-function. Moreover, it is possible to calculate the TW-function $\boldsymbol{M}_{\text {ker }}(\lambda)$ associated with the kernel of the graph. Thus it remains to prove that the kernel of the graph and the potential on it can be reconstructed from $\boldsymbol{M}_{\mathrm{ker}}(\lambda)$.

Reconstruction of the graph is a relatively simple problem, since the TW-function $\boldsymbol{M}_{\mathrm{ker}}(\lambda)$ allows one to calculate the lengths of the shortest paths connecting the boundary points (by considering the wave equation evolution on the graph and the corresponding dynamical response operators, which are in one-to-one correspondence with the TW-function $[24,11])$. Moreover, zeroes of the TW-function determine the spectrum of the Schrödinger operator on the cycle without any contact points, i.e. defined on the functions which are continuous and have continuous derivatives at the vertices $V_{1}, V_{2}$, and $V_{3}$. Therefore the total length of the cycle is also determined by $\boldsymbol{M}_{\mathrm{ker}}(\lambda)$. This information (the lengths of the shortest paths and the total length) is enough to reconstruct the kernel of $\Gamma$.

Reconstruction of the potential is a slightly more sophisticated problem. To determine the potential it is enough to know the functions $t_{12}^{m}$ and $t_{11}^{m}, m=1,2,3$ [36-39]. (Their zeroes determine the Dirichlet-Dirichlet and Neumann-Dirichlet spectra of the corresponding Sturm-Liouville operators.) Our method to obtain these functions is closely related to the solution of the inverse problem for periodic one-dimensional Schrödinger operator developed by Marchenko and Ostrovsky [33, 34], see also [32] and [40]. It is clear that $\boldsymbol{M}_{\mathrm{ker}}(\lambda)$ determines uniquely the following functions:

$$
t_{12}^{1}, t_{12}^{2}, t_{12}^{3},\left(T^{1} T^{3}\right)_{12},\left(T^{2} T^{1}\right)_{12},\left(T^{3} T^{2}\right)_{12} .
$$

Since the functions $t_{12}^{m}$ are already determined, in order to determine potentials on the intervals $\left[x_{2 m-1}, x_{2 m}\right]$ it remains to calculate the functions $t_{11}^{m}$. These functions are entire functions of exponential type not larger than $x_{2 m}-x_{2 m-1}$ (considered as functions of the spectral parameter $\left.k, k^{2}=\lambda\right)$.

As an example let us discuss how to calculate the function $t_{11}^{1}$. The function

$$
\left(T^{1} T^{3}\right)_{12}=t_{11}^{1} t_{12}^{3}+t_{12}^{1} t_{22}^{3}
$$

is determined by the element $\left(\boldsymbol{M}_{\mathrm{ker}}(\lambda)\right)_{11}$. Consider the zeroes of the function $t_{12}^{1}$ to be denoted by $k_{j}^{1}$, so that the numbers $\left(k_{j}^{1}\right)^{2}=\lambda_{j}^{1}$ form the Dirichlet-Dirichlet spectrum on $\left[x_{1}, x_{2}\right]$. If $t_{12}^{3}\left(k_{j}^{1}\right) \neq 0$, then the value of the function $t_{11}^{1}$ at this point can be calculated 
$t_{11}^{1}\left(k_{j}^{1}\right)=\left(T^{1} T^{3}\right)_{12}\left(k_{j}^{1}\right) / t_{12}^{3}\left(k_{j}^{1}\right)$.

If $t_{12}^{3}\left(k_{j}^{1}\right)=0$, then consider the function

$$
\left(T^{2} T^{1}\right)_{12}=t_{11}^{2} t_{12}^{1}+t_{12}^{2} t_{22}^{1}
$$

determined by $\left(\mathbf{M}_{\mathrm{ker}}(\lambda)\right)_{22}$. Note that $t_{12}^{2}\left(k_{j}^{1}\right) \neq 0$, since otherwise the system would have a resonance with the energy $E=\left(k_{j}^{1}\right)^{2}$. Taking into account that the transfer matrix has unit determinant we may calculate the value of $t_{11}^{1}\left(k_{j}^{1}\right)$ :

$$
t_{11}^{1}\left(k_{j}^{1}\right)=\frac{1}{t_{22}^{1}\left(k_{j}^{1}\right)}=\frac{t_{12}^{2}\left(k_{j}^{1}\right)}{\left(T^{2} T^{1}\right)_{12}\left(k_{j}^{1}\right)} .
$$

Summing up we have calculated the values $t_{11}^{1}\left(k_{j}^{1}\right)$ for all $j$, but the points $k_{j}^{1}$ satisfy the standard asymptotics

$$
k_{j}^{1}=\frac{\pi j}{x_{2}-x_{1}}+\mathrm{O}(1) .
$$

Hence the entire function $t_{11}^{1}$ is uniquely determined by its values at these points (see e.g. [41]).

The two other functions $t_{11}^{2}$ and $t_{11}^{3}$ can be reconstructed in a similar way. Thus the functions $t_{11}^{m}$ and $t_{12}^{m}$ are reconstructed, which allows one to calculate the unique potential $q$ on $\operatorname{ker}(\Gamma)$.

The theorem implies that for almost all potentials the inverse problem has a unique solution, since generically the resonance is absent. In principle the non-resonance condition can be weakened, but as will be shown in the following section, it is impossible to get rid of a certain restricting condition completely. The proof of the theorem shows that even in the case of resonance the kernel of the graph can be reconstructed uniquely.

\section{Remark on magnetic Schrödinger operators}

One may try to consider the same procedure as the one developed in [11] for cycles with just two contact points by considering external magnetic fields and thus using spectral data for different values of the magnetic flux through the cycle. Consider the differential operator

$$
L_{q, a}=\left[-\frac{1}{\mathrm{i}} \frac{\mathrm{d}}{\mathrm{d} x}+a(x)\right]^{2}+q(x),
$$

where $a \in C(\Gamma)$ and $q \in L_{2}(\Gamma)$ are real magnetic and (electric) potentials, respectively. Let us denote by $\Phi_{j}$ the integrals of the magnetic potential $a$ along the intervals $\left[x_{2 j-1}, x_{2 j}\right]$ :

$$
\Phi_{j}=\int_{x_{2 j-1}}^{x_{2 j}} a(x) \mathrm{d} x .
$$

The corresponding TW-function is given by

$$
\boldsymbol{M}_{\mathrm{ker}}(\lambda)=\left(\begin{array}{ccc}
-\frac{t_{11}^{1} t_{12}^{3}+t_{12}^{1} t_{22}^{3}}{t_{12}^{1} t_{12}^{3}} & \frac{\mathrm{e}^{-\mathrm{i} \Phi_{1}}}{t_{12}^{1}} & \frac{\mathrm{e}^{\mathrm{i} \Phi_{3}}}{t_{12}^{3}} \\
\frac{\mathrm{e}^{\mathrm{i} \Phi_{1}}}{t_{12}^{1}} & -\frac{t_{11}^{2} t_{12}^{1}+t_{12}^{2} t_{22}^{1}}{t_{12}^{2} t_{12}^{1}} & \frac{\mathrm{e}^{-\mathrm{i} \Phi_{2}}}{t_{12}^{2}} \\
\frac{\mathrm{e}^{-\mathrm{i} \Phi_{3}}}{t_{12}^{3}} & \frac{\mathrm{e}^{\mathrm{i} \Phi_{1} 2}}{t_{12}^{2}} & -\frac{t_{11}^{3} t_{12}^{2}+t_{12}^{3} t_{22}^{2}}{t_{12}^{3} t_{12}^{2}}
\end{array}\right)
$$

One can see that considering spectral data depending on the magnetic flux does not help us to obtain any new function in addition to the list given in (4.6). On the other hand, the phases $\Phi_{j}$ can easily be reconstructed from (5.2) (up to $2 \pi k, k \in \mathbb{Z}$ ) and therefore all possible information concerning the magnetic potential is uniquely determined by $\boldsymbol{M}_{\mathrm{ker}}(\lambda)$. (The magnetic Schrödinger operators with equal electric potentials $q$ and the same phases $\Phi_{j}$ are unitary equivalent.)

\section{Counterexample}

In this section we are going to present a counterexample showing that in general $\boldsymbol{M}_{\mathrm{ker}}(\lambda)$ does not determine a unique potential on $\operatorname{ker} \Gamma$, in other words, that certain conditions that guarantee uniqueness are really needed.
This counterexample is a modification of the example presented in [11].

Consider the case where $\operatorname{ker} \Gamma$ is a ring divided into three equal intervals, i.e. $x_{2}-x_{1}=x_{4}-x_{3}=x_{6}-x_{5}$. In addition, we assume that the corresponding potentials have transfer matrices with equal traces and elements 12 :

$$
\begin{gathered}
t_{12}^{1}=t_{12}^{2}=t_{12}^{3}:=t_{12}, \\
t_{11}^{1}+t_{22}^{1}=t_{11}^{2}+t_{22}^{2}=t_{11}^{3}+t_{22}^{3}:=2 u_{+} \cdot \\
\text { Introducing notations } \\
\quad u_{-}^{m}=\left(t_{11}^{m}-t_{22}^{m}\right) / 2, \quad m=1,2,3,
\end{gathered}
$$

the corresponding TW-function can be written in the form 


$$
\boldsymbol{M}_{\mathrm{ker}}(\lambda)=\frac{1}{t_{12}}\left(\begin{array}{ccc}
-\left(u_{-}^{1}-u_{-}^{3}+2 u_{+}\right) & 1 & 1 \\
1 & -\left(u_{-}^{2}-u_{-}^{1}+2 u_{+}\right) & 1 \\
1 & 1 & -\left(u_{-}^{3}-u_{-}^{2}+2 u_{+}\right)
\end{array}\right) \text {. }
$$

The matrix function $\boldsymbol{M}_{\mathrm{ker}}(\lambda)$ allows one to reconstruct the following functions:

$$
\begin{aligned}
& t_{12}=1 /\left(\boldsymbol{M}_{\mathrm{ker}}(\lambda)\right)_{12}, \\
& u_{+}=-t_{12} \operatorname{Tr} \boldsymbol{M}_{\mathrm{ker}}(\lambda) / 6, \\
& u_{-}^{m}-u_{-}^{m-1}=-t_{12} M_{m m}(\lambda)-2 u_{+}, \\
& \quad m=1,2,3,
\end{aligned}
$$

(with the indices 0 and 3 identified). Since the entries of $\boldsymbol{M}_{\mathrm{ker}}(\lambda)$ depend only on the listed functions no other characteristics of the system may be calculated.

Knowledge of $u_{+}$and $t_{12}$ is not enough to reconstruct potentials on the corresponding intervals. Consider zeroes $k_{j}$ of the function $t_{12}$. It appears that the values of $u_{-}^{m}$ at these points are determined up to the signs $\nu_{j}^{m}$ :

$$
u_{-}^{m}\left(k_{j}\right)=\nu_{j}^{m} \sqrt{u_{+}^{2}\left(k_{j}\right)-1} .
$$

Hence, provided the functions $t_{12}$ and $u_{+}$are known, the function $u_{-}^{m}$ is uniquely determined by the sequence of signs $\left\{\nu_{j}^{m}\right\}, \nu_{j}^{m}= \pm 1$.

Consider the case where $\nu_{j}^{1}=\nu_{j}^{2}=\nu_{j}^{3}$ for a certain $j$. These signs may be changed to the opposite ones and this transformation does not affect the functions $u_{-}^{m}-u_{-}^{m-1}$. It is clear that such modification of the functions $u_{-}^{m}$ leads to a different potential. One may obtain discrete families of isomorphic potentials by changing several equal indices.

\section{Conclusions}

It is interesting to compare these results with [11], where the case of one cycle with just one and two contact points was considered. It appears that in some sense it becomes "easier" to reconstruct potential as the number of vertices on the cycle increases. In the case of one vertex this reconstruction in general is not unique. If the number of vertices is equal to two, then considering spectral data for different values of the magnetic flux through the cycle, one may reconstruct the potential, provided there is no resonance. In the case of three vertices, to reconstruct the potential it is enough to know the TW-function for just one value of the magnetic flux.

It is clear that all phenomena discussed here are present in the case of graphs with one cycle and several (more than three) contact points as well as for graphs having more than one cycle. The results of these investigations will be presented in one of the forthcoming publications. It is clear that the notion of the resonance discussed here is going to play a crucial role in these investigations.

\section{Acknowledgments}

The author was supported in part by Swedish Research Council grant No. 50092501.

\section{References}

[1] S. Albeverio, P. Kurasov, Singular Perturbations of Differential Operators. Solvable Schrödinger Type Operators, London Mathematical Society Lecture Note Series, Vol. 271, Cambridge University Press, Cambridge, 2000.

[2] Quantum Graphs and Their Applications. Proc. AMS-IMS-SIAM Joint Summer Research Conf., Snowbird, UT, 2005, Eds. G. Berkolaiko, R. Carlson, S.A. Fulling, P. Kuchment, Contemporary Mathematics, Vol. 415, American Mathematical Society, 2006.

[3] P. Kuchment, Waves Random Media 14, S107, (2004).

[4] P. Kuchment, J. Phys. A 38, 4887 (2005).

[5] Yu.V. Pokornyi, O.M. Penkin, V.L. Pryadiev, A.V. Borovskikh, K.P. Lazarev, S.A. Shabrov, Differential Equations on Geometric Graphs, Fiziko-Matematicheskaya Literatura, Moscow 2005 (in Russian).

[6] J. von Below, in: Partial Differential Equations on Multistructures, Luminy, 1999, Lecture Notes in Pure and Appl. Math., Vol. 219, Dekker, New York 2001, p. 19.

[7] J. Boman, P. Kurasov, Adv. Appl. Math. 35, 58 (2005).

[8] B. Gutkin, U. Smilansky, J. Phys. A 34, 6061 (2001).

[9] P. Kurasov, M. Nowaczyk, J. Phys. A 38, 4901 (2005).

[10] P. Kurasov, F. Stenberg, J. Phys. A 35, 101 (2002).

[11] P. Kurasov, accepted for publication in Math. Proc. Camb. Phil. Soc.

[12] M. Harmer, J. Phys. A 38, 4875 (2005).

[13] V. Kostrykin, R. Schrader, J. Phys. A 32, 595 (1999).

[14] V. Kostrykin, R. Schrader, Fortschr. Phys. 48, 703 (2000).

[15] V. Kostrykin, R. Schrader, The inverse scattering problem for metric graphs and the travelling salesman problem, arXiv:math-ph/0603010.

[16] N.I. Gerasimenko, B.S. Pavlov, Theoret. Math. Phys. 74, 230 (1988).

[17] N.I. Gerasimenko, Theoret. Math. Phys. 75, 460 (1988).

[18] M.I. Belishev, Inverse Problems 20, 647 (2004).

[19] M.I. Belishev, J. Math. Sci. (New York) 132, 11 (2006).

[20] M.I. Belishev, A.F. Vakulenko, J. Inverse Ill-Posed Probl. 14, 29 (2006). 
[21] B.M. Brown, R. Weikard, Proc. R. Soc. Lond. Ser. A Math. Phys. Eng. Sci. 461, 3231 (2005).

[22] V. Yurko, Math. Notes 79, 572 (2006).

[23] V. Yurko, Inverse Problems 21, 1075 (2005).

[24] S. Avdonin, P. Kurasov, Inverse Probl. Imaging 2, 1 (2008).

[25] F. Gesztesy, E. Tsekanovskii, Math. Nachr. 218, 61 (2000).

[26] Yu. I. Lyubarskiı̆, V.A. Marchenko, Funktsional. Anal. i Prilozhen. 41, 58 (2007).

[27] M.I. Belishev, Inverse Problems 23, R1 (2007).

[28] V. Pivovarchik, Oper. Theory Adv. Appl. 124, 527 (2001).

[29] V. Yurko, Operators Matrices 2, 543 (2008).

[30] J. Garnett, E. Trubowitz, Comment. Math. Helv. 59 258 (1984).

[31] J. Garnett, E. Trubowitz, Comment. Math. Helv. 62 18 (1987).
[32] H.P. McKean, E. Trubowitz, Commun. Pure Appl. Math. 29, 143 (1976)

[33] V.A. Marchenko, I.V. Ostrovsky, Mat. Sb. (N.S.) 97 (139), 540 (1975).

[34] V.A. Marchenko, I.V. Ostrovsky, Selecta Matematica Sovietica 6, 101 (1987).

[35] S. Avdonin, V. Mikhaylov, A. Rybkin, Commun. Math. Phys. 275, 791 (2005).

[36] G. Borg, Acta Math. 78, 1 (1946).

[37] G. Borg, in: Den 11te Skandinaviske Matematikerkongress, Trondheim, 1949, p. 276.

[38] N. Levinson, Danske Vid. Selsk. Mat.-Fys. Medd. 25, 29 (1949).

[39] B.M. Levitan, M.G. Gasymov, Uspekhi Mat. Nauk 19, 116, 3 (1964).

[40] J. Bourgain, Geom. Func. Anal. 3, 209 (1993).

[41] B.Ya. Levin, Lectures on Entire Functions, AMS, 1996. 